\title{
Physics
}

\section{Bi-Hamiltonian Structure of a Three-Component Camassa-Holm Type Equation}

Nianhua Li, Q.P. Liu

To cite this article: Nianhua Li, Q.P. Liu (2013) Bi-Hamiltonian Structure of a Three-

Component Camassa-Holm Type Equation, Journal of Nonlinear Mathematical Physics 20:1, 126-134, DOI: https://doi.org/10.1080/14029251.2013.792477

To link to this article: https://doi.org/10.1080/14029251.2013.792477

Published online: 04 January 2021 


\title{
Bi-Hamiltonian Structure of a Three-Component Camassa-Holm Type Equation
}

\author{
Nianhua Li and Q.P. Liu \\ Department of Mathematics \\ China University of Mining and Technology \\ Beijing, 100083, P. R. China \\ linianhua1984@sina.com.cn, qpl@cumtb.edu.cn \\ Received 9 September 2012 \\ Accepted 22 January 2012
}

\begin{abstract}
A recently proposed three-component Camassa-Holm equation is considered. It is shown that this system is a bi-Hamiltonian system.

Keywords: bi-Hamiltonian structures; three-component $\mathrm{CH}$ equation

2000 Mathematics Subject Classification: 37K10, 35Q51, 35Q58
\end{abstract}

\section{Introduction}

In 1981, the following integrable equation

$$
u_{t}-u_{x x t}+3 u u_{x}=2 u_{x} u_{x x}+u u_{x x x}
$$

was proposed as an abstract equation admitting a bi-Hamiltonian structure [20]. This system, now known as Camassa-Holm (CH) equation, was rediscovered by Camassa and Holm [3] as an approximation to the incompressible Euler equations and its relevance for the shallow water waves modeling was discussed [14,24-26]. The $\mathrm{CH}$ equation has been studied extensively and a number of properties are established for it. Indeed, it is shown that this equation is solvable by inverse scattering transformation $[1,2,5,6,15,17,27,30]$, possesses a large number of solutions including multi-soliton solutions, algebraic-geometric solutions and multi-peakon solutions [22, 33, 34, 37], can be regarded as the geodesic flow on the diffeomorphism group [29,35] (see also [12, 13,28]), to mention just a few (also see [21,32] and the references there).

Also, it is worthwhile to remark that while the $\mathrm{CH}$ equation is reciprocally associated to a particular flow of the celebrated Korteweg-de Vries (KdV) equation [19,32], there are fundamental differences between the $\mathrm{CH}$ equation and the $\mathrm{KdV}$ equation. Unlike the $\mathrm{KdV}$ equation, the $\mathrm{CH}$ equation has breaking wave solutions which develop singularities in finite time [8]. Indeed, the construction of such an equation, which can model both breaking waves and peaked traveling waves, was a long-standing open problem in hydrodynamics [39]. Furthermore, the search of models which have peaked wave solutions is also motivated by the quest for waves of great height (see $[7,9,10$, 38]). It is interesting to note that the peaked solitons or peakons of the $\mathrm{CH}$ equation are orbitally stable $[16,31]$. 
The $\mathrm{CH}$ equation has several generalizations. A two-component and a three-component extensions were proposed in $[4,36]$ and in [18] respectively and both extensions are shown to be biHamiltonian systems. Very recently, a new three-component generalization of the $\mathrm{CH}$ equation was constructed by Geng and Xue [21]. It reads as

$$
\begin{aligned}
u_{t} & =-v p_{x}+u_{x} q+\frac{3}{2} u q_{x}-\frac{3}{2} u\left(p_{x} r_{x}-p r\right), \\
v_{t} & =2 v q_{x}+v_{x} q, \\
w_{t} & =v r_{x}+w_{x} q+\frac{3}{2} w q_{x}+\frac{3}{2} w\left(p_{x} r_{x}-p r\right),
\end{aligned}
$$

with

$$
\begin{aligned}
u & =p-p_{x x}, \\
v & =\frac{1}{2}\left(q_{x x}-4 q+p_{x x} r_{x}-r_{x x} p_{x}+3 p_{x} r-3 p r_{x}\right), \\
w & =r_{x x}-r .
\end{aligned}
$$

Above system was derived from a $3 \times 3$ matrix spectral problem. It was further shown that (1.2) is a Hamiltonian system with infinite number of conservation laws. The purpose of this paper is to construct two Hamiltonian structures for (1.2) and to prove that it constitutes a bi-Hamiltonian system.

\section{Bi-Hamiltonian structure of the three-component Camassa-Holm equation}

As demonstrated in [21], the system (1.2) arises as a zero curvature equation

$$
M_{t}-N_{x}+[M, N]=0,
$$

which is the compatibility condition of the linear system

$$
\varphi_{x}=M \varphi, \quad \varphi_{t}=N \varphi .
$$

where

$$
\begin{aligned}
M & =\left(\begin{array}{ccc}
0 & 1 & 0 \\
1+\lambda v & 0 & u \\
\lambda w & 0 & 0
\end{array}\right), \\
N & =\left(\begin{array}{ccc}
\frac{1}{2}\left(-q_{x}-p_{x} r_{x}+p r\right) & \frac{1}{\lambda}+q & \frac{p_{x}}{\lambda} \\
\frac{1}{\lambda}-p r_{x}+p_{x} r-q+\lambda q v & \frac{1}{2}\left(q_{x}-p_{x} r_{x}+p r\right) & \frac{p}{\lambda}+q u \\
-r+\lambda q w & r_{x} & p_{x} r_{x}-p r
\end{array}\right) .
\end{aligned}
$$

Now, we construct Hamiltonian structures for the equations associated with this $3 \times 3$ spectral problem. To this end, we take $M$ as above and $N$ as follows

$$
N=\left(\begin{array}{lll}
A_{1} & A_{2} & A_{3} \\
B_{1} & B_{2} & B_{3} \\
C_{1} & C_{2} & C_{3}
\end{array}\right),
$$

with $A_{k}, B_{k}, C_{k}(k=1,2,3)$ are functions depending on the spectral parameter $\lambda$ and the field variables. 
Through tedious calculations, we find that the zero-curvature representation (2.1) yields

$$
\left(\begin{array}{c}
u \\
v \\
w
\end{array}\right)_{t}=\left(\lambda^{-1} \mathscr{E}+\mathscr{F}\right)\left(\begin{array}{c}
C_{2} \\
A_{2} \\
\lambda A_{3}
\end{array}\right)
$$

where

$$
\begin{aligned}
& \mathscr{E}=\left(\begin{array}{ccc}
0 & 0 & \partial^{2}-1 \\
\frac{1}{2} u_{x}+\frac{3}{2} u \partial & 2 \partial-\frac{1}{2} \partial^{3} & \frac{1}{2} w_{x}+\frac{3}{2} w \partial \\
1-\partial^{2} & 0 & 0
\end{array}\right), \\
& \mathscr{F}=\left(\begin{array}{ccc}
\frac{3}{2} u \partial^{-1} u & u_{x}+\frac{3}{2} u \partial & -\frac{3}{2} u \partial^{-1} w-v \\
0 & v \partial+\partial v & 0 \\
-\frac{3}{2} w \partial^{-1} u+v w_{x}+\frac{3}{2} w \partial & \frac{3}{2} w \partial^{-1} w
\end{array}\right) .
\end{aligned}
$$

While neither $\mathscr{E}$ nor $\mathscr{F}$ could serve as Hamiltonian operators since both of them are even not skewsymmetric, we may formulate a operator

$$
\mathscr{R}=\mathscr{F} \mathscr{E}^{-1},
$$

which is the candidate for a recursion operator. To justify this, we notice that a Hamiltonian operator was obtained in [21], which reads as

$$
\mathscr{J}=\left(\begin{array}{ccc}
0 & 0 & \partial^{2}-1 \\
0 & -\partial v-v \partial & 0 \\
1-\partial^{2} & 0 & 0
\end{array}\right)
$$

Then we define a new operator by

$$
\mathscr{K}=\mathscr{F} \mathscr{E}^{-1} \mathscr{J}
$$

whose explicit form is given by

$$
\mathscr{K}=\mathscr{T}-2 \mathscr{S}\left(\partial^{3}-4 \partial\right)^{-1}\left(\mathscr{S}^{\dagger}\right)
$$

with

$$
\mathscr{S}=\left(\begin{array}{c}
\frac{3}{2} u \partial+u_{x} \\
v \partial+\partial v \\
\frac{3}{2} w \partial+w_{x}
\end{array}\right), \quad \mathscr{T}=\left(\begin{array}{ccc}
\frac{3}{2} u \partial^{-1} u & 0-v-\frac{3}{2} u \partial^{-1} w \\
0 & 0 & 0 \\
v-\frac{3}{2} w \partial^{-1} u & 0 & \frac{3}{2} w \partial^{-1} w
\end{array}\right)
$$

and $\mathscr{S}^{\dagger}$ denotes the formal adjoint of the operator $\mathscr{S}$.

Our claim is that $\mathscr{J}$ and $\mathscr{K}$ constitutes a compatible Hamiltonian pair. In fact, we have 
N.H. Li \& Q.P. Liu

Theorem 2.1. The system (1.2) is a bi-Hamiltonian system, namely there exist two compatible Hamiltonian operators $\mathscr{J}$ and $\mathscr{K}$ such that

$$
\left(\begin{array}{c}
u \\
v \\
w
\end{array}\right)_{t}=\mathscr{J}\left(\begin{array}{c}
\frac{\delta H_{1}}{\delta u} \\
\frac{\delta H_{1}}{\delta v} \\
\frac{\delta H_{1}}{\delta w}
\end{array}\right)=\mathscr{K}\left(\begin{array}{c}
\frac{\delta H_{0}}{\delta u} \\
\frac{\delta H_{0}}{\delta v} \\
\frac{\delta H_{0}}{\delta w}
\end{array}\right),
$$

where $\mathscr{J}$ and $\mathscr{K}$ are the operators given by (2.8) and (2.9), and

$$
\begin{aligned}
& H_{0}=\frac{1}{4} \int\left(4 q^{2}-q q_{x x}-p_{x}^{2} r_{x}^{2}+6 p p_{x} r r_{x}+3 p^{2} r^{2}\right) d x, \\
& H_{1}=\frac{1}{2} \int\left(2 v+u r_{x}+w p_{x}\right) d x .
\end{aligned}
$$

Proof. The first operator $\mathscr{J}$ is a local and linear operator, which already appeared in [21]. Next, we turn to the more complicated operator $\mathscr{K}$. Also, it is easy to show that this operator is skewsymmetric, therefore we prove that the related Jacobi identity holds. Since

$$
\begin{aligned}
& <f, \mathscr{K}^{\prime}[\mathscr{K} g] h>+ \text { c.p. } \\
& =<f, \mathscr{T}^{\prime}[\mathscr{K} g] h-2 \mathscr{S}^{\prime}[\mathscr{K} g]\left(\partial^{3}-4 \partial\right)^{-1}\left(\mathscr{S}^{\dagger}\right) h-2 \mathscr{S}\left(\partial^{3}-4 \partial\right)^{-1}\left(\mathscr{S}^{\dagger}\right)^{\prime}[\mathscr{K} g] h>+ \text { c.p.. }
\end{aligned}
$$

Let $A, B, C$ be the rows of $\mathscr{K}$, or $\mathscr{K}=(A, B, C)^{\mathrm{T}}$, we find

$$
\begin{aligned}
< & f, \mathscr{T}^{\prime}[\mathscr{K} g] h>+ \text { c.p. } \\
= & <\left(\begin{array}{c}
f_{1} \\
f_{2} \\
f_{3}
\end{array}\right),\left(\begin{array}{c}
\frac{3}{2} A g \partial^{-1}\left(u h_{1}-w h_{3}\right)+\frac{3}{2} u \partial^{-1} A g h_{1}-B g h_{3}-\frac{3}{2} u \partial^{-1} C g h_{3} \\
0 \\
B g h_{1}-\frac{3}{2} C g \partial^{-1}\left(u h_{1}-w h_{3}\right)-\frac{3}{2} w \partial^{-1} A g h_{1}+\frac{3}{2} w \partial^{-1} C g h_{3}
\end{array}\right)>+ \text { c.p. } \\
= & <\frac{3}{2}\left(f_{1} A g \partial^{-1}\left(u h_{1}-w h_{3}\right)+u f_{1} \partial^{-1} A g h_{1}-u f_{1} \partial^{-1} C g h_{3}\right)-f_{1} B g h_{3}> \\
& +<\frac{3}{2}\left(-w f_{3} \partial^{-1} h_{1} A g-f_{3} C g \partial^{-1}\left(u h_{1}-w h_{3}\right)+w f_{3} \partial^{-1} C g h_{3}\right)+f_{3} B g h_{1}>+ \text { c.p. } \\
= & <\frac{3}{2}\left(f_{1} A g-g_{1} A f+C f g_{3}-f_{3} C g\right) \partial^{-1}\left(u h_{1}-w h_{3}\right)+f_{3} B g h_{1}-f_{1} B g h_{3}>+ \text { c.p. } \\
= & \frac{9}{4}\left(<u g_{1} \partial^{-1} w f_{3}+w g_{3} \partial^{-1} w f_{3}, \partial^{-1} u h_{1}>+<u g_{1} \partial^{-1} u f_{1}+w g_{3} \partial^{-1} u f_{1}, \partial^{-1} h_{3} w>\right) \\
& +3<f_{1}\left(\frac{3}{2} u \partial+u_{x}\right) g_{0}-f_{3}\left(\frac{3}{2} w \partial+w_{x}\right) g_{0}, \partial^{-1}\left(u h_{1}-w h_{3}\right)> \\
& +2<\left(v_{x}+2 v \partial\right) h_{0}, f_{1} g_{3}>-(f \leftrightarrow g)+\text { c.p. } \\
= & 3<\left(\frac{3}{2} f_{1} u \partial+f_{1} u_{x}\right) g_{0}-\left(\frac{3}{2} f_{3} w \partial+f_{3} w_{x}\right) g_{0}, \partial^{-1}\left(u h_{1}-h_{3} w\right)> \\
& +2<\left(v_{x}+2 v \partial\right) h_{0}, f_{1} g_{3}>-(f \leftrightarrow g)+\text { c.p. } \\
= & \frac{3}{2}<g_{0},\left(f_{3} w_{x}+3 f_{3 x} w-f_{1} u_{x}-3 f_{1 x} u\right) \partial^{-1}\left(u h_{1}-w h_{3}\right)> \\
& +2<g_{0}, v_{x} f_{1} h_{3}+2 v\left(f_{1} h_{3}\right)_{x}>-(f \leftrightarrow h)+\text { c.p. },
\end{aligned}
$$

where $(f \leftrightarrow g)$ denotes the resulted term from the previous ones by permutating $f$ and $g$, and

$$
g_{0} \equiv\left(\partial^{3}-4 \partial\right)^{-1}\left(\frac{3}{2} u g_{1 x}+\frac{1}{2} u_{x} g_{1}+v_{x} g_{2}+2 v g_{2 x}+\frac{3}{2} w g_{3 x}+\frac{1}{2} w_{x} g_{3}\right),
$$


and similarly for $f_{0}, h_{0}$. Also, we have

$$
\begin{aligned}
< & f,-2 \mathscr{S}^{\prime}[\mathscr{K} g]\left(\partial^{3}-4 \partial\right)^{-1}\left(\mathscr{S}^{\dagger}\right) h-2 \mathscr{S}\left(\partial^{3}-4 \partial\right)^{-1}\left(\mathscr{S}^{\dagger}\right)^{\prime}[\mathscr{K} g] h>+ \text { c.p. } \\
= & 2<h, \mathscr{S}^{\prime}[\mathscr{K} f] g_{0}>-2<h, \mathscr{S}^{\prime}[\mathscr{K} g] f_{0}>+ \text { c.p. } \\
= & 2<h^{T} \mathscr{S}^{\prime}[\mathscr{K} f] g_{0}-f^{T} \mathscr{S}^{\prime}[\mathscr{K} h] g_{0}>+ \text { c.p. } \\
= & 2<\left(\frac{3}{2} h_{1} A f \partial+h_{1}(A f)_{x}+h_{2}\left((B f)_{x}+2 B f \partial\right)+h_{3}\left(\frac{3}{2} C f \partial+(C f)_{x}\right)\right) g_{0}> \\
& -(f \leftrightarrow h)+\text { c.p. } \\
= & <g_{0}, f_{1}(A h)_{x}+3 f_{1 x} A h+2 f_{2}(B h)_{x}+4 f_{2 x} B h+f_{3}(C h)_{x}+3 f_{3 x} C h> \\
& -(f \leftrightarrow h)+\text { c.p. } \\
\equiv & \Omega_{1}+\Omega_{2},
\end{aligned}
$$

where

$$
\begin{aligned}
\Omega_{1} & =<g_{0}, f_{1}\left(\frac{3}{2} u_{x} \partial^{-1}\left(u h_{1}-w h_{3}\right)+\frac{3}{2} u^{2} h_{1}-v_{x} h_{3}-v h_{3 x}-\frac{3}{2} u w h_{3}\right)> \\
& +<g_{0}, 3 f_{1 x}\left(\frac{3}{2} u \partial^{-1}\left(u h_{1}-w h_{3}\right)-v h_{3}\right)+3 f_{3 x}\left(v h_{1}-\frac{3}{2} w \partial^{-1}\left(u h_{1}-w h_{3}\right)\right)> \\
& +<g_{0}, f_{3}\left(v_{x} h_{1}+v h_{1 x}-\frac{3}{2} w_{x} \partial^{-1}\left(u h_{1}-w h_{3}\right)-\frac{3}{2} w u h_{1}+\frac{3}{2} w^{2} h_{3}\right)> \\
& -(f \leftrightarrow h)+\text { c.p. } \\
= & \frac{3}{2}<g_{0},\left(f_{1} u_{x}+3 f_{1 x} u-f_{3} w_{x}-3 f_{3 x} w\right) \partial^{-1}\left(u h_{1}-w h_{3}\right)> \\
& +<g_{0}, 3 h_{1 x} v f_{3}-h_{1}\left(\frac{3}{2} u^{2} f_{1}-v_{x} f_{3}-v f_{3 x}-\frac{3}{2} u w f_{3}\right)> \\
& -<g_{0}, 3 h_{3 x} v f_{1}+h_{3}\left(v_{x} f_{1}+v f_{1 x}-\frac{3}{2} u w f_{1}+\frac{3}{2} w^{2} f_{3}\right)>-(f \leftrightarrow h)+\text { c.p. } \\
= & \frac{3}{2}<g_{0},\left(f_{1} u_{x}+3 f_{1 x} u-f_{3} w_{x}-3 f_{3 x} w\right) \partial^{-1}\left(u h_{1}-w h_{3}\right)> \\
& +2<g_{0}, v_{x}\left(f_{3} h_{1}\right)+2 v\left(f_{3 x} h_{1}+f_{3} h_{1 x}\right)>-(f \leftrightarrow h)+\text { c.p. },
\end{aligned}
$$

and

$$
\begin{aligned}
\Omega_{2}= & 2<g_{0},\left(f_{1} \partial+3 f_{1 x}\right)\left(\frac{3}{2} u \partial+u_{x}\right) h_{0}+\left(2 f_{2} \partial+4 f_{2 x}\right)\left(v_{x}+2 v \partial\right) h_{0}> \\
& +2<g_{0},\left(f_{3} \partial+3 f_{3 x}\right)\left(\frac{3}{2} w \partial+w_{x}\right) h_{0}>-(f \leftrightarrow h)+\text { c.p.. }
\end{aligned}
$$

Taking account of (2.12)-(2.14) with (2.15)-(2.16), we obtain

$$
\begin{aligned}
< & f, \mathscr{K}^{\prime}[\mathscr{K} g] h>+ \text { c.p. }=\Omega_{2} \\
= & 2<g_{0},\left(f_{1}\left(\frac{3}{2} u \partial^{2}+\frac{5}{2} u_{x} \partial+u_{x x}\right)+3 f_{1 x}\left(\frac{3}{2} u \partial+u_{x}\right)\right) h_{0}> \\
& +2<g_{0},\left(f_{3}\left(\frac{3}{2} w \partial^{2}+\frac{5}{2} w_{x} \partial+w_{x x}\right)+3 f_{3 x}\left(\frac{3}{2} w \partial+w_{x}\right)\right) h_{0}> \\
& \left.+4<g_{0}, f_{2}\left(v_{x x}+3 v_{x} \partial+2 v \partial^{2}\right)+2 f_{2 x}\left(v_{x}+2 v \partial\right)\right) h_{0}>-(f \leftrightarrow h)+\text { c.p. }
\end{aligned}
$$


N.H. Li \& Q.P. Liu

$$
\begin{aligned}
= & 2<h_{0},\left(\frac{3}{2} \partial^{2} f_{1} u-\frac{5}{2} \partial f_{1} u_{x}+u_{x x} f_{1}-\frac{9}{2} \partial u f_{1 x}+3 f_{1 x} u_{x}\right) g_{0}> \\
& +2<h_{0},\left(\frac{3}{2} \partial^{2} w f_{3}-\frac{5}{2} \partial f_{3} w_{x}+w_{x x} f_{3}-\frac{9}{2} \partial w f_{3 x}+3 f_{3 x} w_{x}\right) g_{0}> \\
& +2<h_{0},\left(2 f_{2} v_{x x}-6 \partial v_{x} f_{2}+4 \partial^{2} f_{2} v+4 f_{2 x} v_{x}-8 \partial f_{2 x} v\right) g_{0}> \\
& -2<g_{0},\left(h_{1}\left(\frac{3}{2} u \partial^{2}+\frac{5}{2} u_{x} \partial+u_{x x}\right)+3 h_{1 x}\left(\frac{3}{2} u \partial+u_{x}\right)\right) f_{0}> \\
& -2<g_{0},\left(h_{3}\left(\frac{3}{2} w \partial^{2}+\frac{5}{2} w_{x} \partial+w_{x x}\right)+3 h_{3 x}\left(\frac{3}{2} w \partial+w_{x}\right)\right) f_{0}> \\
& -2<g_{0},\left(2 h_{2}\left(v_{x x}+3 v_{x} \partial+2 v \partial^{2}\right)+4 h_{2 x}\left(v_{x}+2 v \partial\right)\right) f_{0}>+\mathrm{c} \cdot \mathrm{p} . \\
= & -2<g_{0}, \partial\left(h_{1} u_{x}+3 h_{1 x} u+2 h_{2} v_{x}+4 h_{2 x} v+w_{x} h_{3}+3 w h_{3 x}\right) f_{0}> \\
& -2<g_{0},\left(h_{1} u_{x}+3 h_{1 x} u+2 h_{2} v_{x}+4 h_{2 x} v+w_{x} h_{3}+3 w h_{3 x}\right) \partial f_{0}>+\mathrm{c} \cdot \mathrm{p} . \\
= & -4<g_{0}, \partial\left(h_{0 x x x}-4 h_{0 x}\right) f_{0}+\left(h_{0 x x x}-4 h_{0 x}\right) \partial f_{0}>+\mathrm{c} \cdot \mathrm{p} . \\
= & -4<\left(h_{0 x x x}-4 h_{0 x}\right)\left(f_{0 x} g_{0}-f_{0} g_{0 x}\right)>+\mathrm{c} \cdot \mathrm{p} . \\
= & -4<h_{0 x x}\left(f_{0 x x} g_{0}-f_{0} g_{0 x x}\right)>+\mathrm{c} \cdot \mathrm{p} . \\
= & 0 .
\end{aligned}
$$

Therefore, $\mathscr{K}$ is a Hamiltonian operator. Finally, we prove that $\mathscr{J}$ and $\mathscr{K}$ form a compatible Hamiltonian pair, that is, we have to show that these operators satisfy the following condition

$$
<f, \mathscr{J}^{\prime}[\mathscr{K} g] h>+<f, \mathscr{K}^{\prime}[\mathscr{J} g] h>+ \text { c.p. }=0
$$

Indeed, by direct calculation, we find

$$
\begin{aligned}
<f, \mathscr{J}^{\prime}[\mathscr{K} g] h>+ \text { c.p. } & =-2<f_{2},\left(\left(v_{x} g_{0}+2 v g_{0 x}\right)_{x}+2\left(v_{x} g_{0}+2 v g_{0 x}\right) \partial\right) h_{2}>+ \text { c.p. } \\
& =2<v_{x} g_{0}+2 v g_{0 x}, h_{2} f_{2 x}-h_{2 x} f_{2}>+ \text { c.p. }
\end{aligned}
$$

and

$$
\begin{aligned}
< & f, \mathscr{K}^{\prime}[\mathscr{J} g] h>+ \text { c.p. } \\
= & <f, \mathscr{J}^{\prime}[\mathscr{J} g] h-2 \mathscr{S}^{\prime}[\mathscr{J} g]\left(\partial^{3}-4 \partial\right)^{-1} \mathscr{S}^{\dagger} h-2 \mathscr{S}^{\dagger}\left(\partial^{3}-4 \partial\right)^{-1}\left(\mathscr{S}^{\dagger}\right)^{\prime}[\mathscr{J} g] h>+ \text { c.p. } \\
= & <f_{1}, \frac{3}{2}\left(g_{3 x x}-g_{3}\right) \partial^{-1}\left(u h_{1}-w h_{3}\right)+\frac{3}{2} u \partial^{-1}\left(\left(g_{3 x x}-g_{3}\right) h_{1}-\left(g_{1}-g_{1 x x}\right) h_{3}\right)> \\
& +<f_{1}, 2 v g_{2 x} h_{3}+v_{x} g_{2} h_{3}>-<f_{3}, 2 v g_{2 x} h_{1}+v_{x} g_{2} h_{1}> \\
& -<f_{3}, \frac{3}{2}\left(g_{1}-g_{1 x x}\right) \partial^{-1}\left(u h_{1}-w h_{3}\right)+\frac{3}{2} w \partial^{-1}\left(\left(g_{3 x x}-g_{3}\right) h_{1}-\left(g_{1}-g_{1 x x}\right) h_{3}\right)> \\
& -2<f, \mathscr{S}^{\prime}[\mathscr{J} g]\left(\partial^{3}-4 \partial\right)^{-1} \mathscr{S}^{\dagger} h+\mathscr{S}\left(\partial^{3}-4 \partial\right)^{-1}\left(\mathscr{S}^{\dagger}\right)^{\prime}[\mathscr{J} g] h> \\
= & <\frac{3}{2}\left(f_{1}\left(g_{3 x x}-g_{3}\right)-f_{3}\left(g_{1}-g_{1 x x}\right)\right) \partial^{-1}\left(u h_{1}-w h_{3}\right)+2 v f_{1} h_{3} g_{2 x}+v_{x} f_{1} g_{2} h_{3}> \\
& -<\frac{3}{2}\left(h_{1}\left(g_{3 x x}-g_{3}\right)-h_{3}\left(g_{1}-g_{1 x x}\right)\right) \partial^{-1}\left(u f_{1}-w f_{3}\right)+2 v f_{3} g_{2 x} h_{1}+f_{3} v_{x} g_{2} h_{1}> \\
& -2<f, \mathscr{S}^{\prime}[\mathscr{J} g]\left(\partial^{3}-4 \partial\right)^{-1} \mathscr{S}^{\dagger} h+\mathscr{S}\left(\partial^{3}-4 \partial\right)^{-1}\left(\mathscr{S}^{\dagger}\right)^{\prime}[\mathscr{J} g] h>
\end{aligned}
$$




$$
\begin{aligned}
= & <\frac{3}{2}\left(f_{1}\left(g_{3 x x}-g_{3}\right)-f_{3}\left(g_{1}-g_{1 x x}\right)\right) \partial^{-1}\left(u h_{1}-w h_{3}\right)+2 v f_{1} h_{3} g_{2 x}+v_{x} f_{1} g_{2} h_{3}> \\
& +2<g_{0}, f^{T}\left(\begin{array}{ccc}
\frac{1}{2} & 0 & 0 \\
0 & 1 & 0 \\
0 & 0 & \frac{1}{2}
\end{array}\right)(\mathscr{K} h)_{x}+f_{x}^{T}\left(\begin{array}{ccc}
\frac{3}{2} & 0 & 0 \\
0 & 2 & 0 \\
0 & 0 & \frac{3}{2}
\end{array}\right) \mathscr{K} h>-(f \leftrightarrow h)+\text { c.p.. }
\end{aligned}
$$

Thus making use of (2.18) with (2.19), we obtain

$$
\begin{aligned}
< & f, \mathscr{K}^{\prime}[\mathscr{J} g] h>+<f, \mathscr{J}^{\prime}[\mathscr{K} g] h>+ \text { c.p. } \\
= & <\frac{3}{2}\left(f_{1}\left(g_{3 x x}-g_{3}\right)-f_{3}\left(g_{1}-g_{1 x x}\right)\right) \partial^{-1}\left(u h_{1}-w h_{3}\right)+2 v f_{1} h_{3} g_{2 x}+v_{x} f_{1} g_{2} h_{3}> \\
& +2<v_{x} g_{0}+2 v g_{0 x}, h_{2} f_{2 x}>+2<g_{0}, f^{T}\left(\begin{array}{ccc}
\frac{1}{2} & 0 & 0 \\
0 & 1 & 0 \\
0 & 0 & \frac{1}{2}
\end{array}\right)(\mathscr{K} h)_{x}+f_{x}^{T}\left(\begin{array}{ccc}
\frac{3}{2} & 0 & 0 \\
0 & 2 & 0 \\
0 & 0 & \frac{3}{2}
\end{array}\right) \mathscr{K} h> \\
& -(f \leftrightarrow h)+\text { c.p. } \\
= & <\frac{3}{2}\left(f_{1}\left(g_{3 x x}-g_{3}\right)-f_{3}\left(g_{1}-g_{1 x x}\right)\right) \partial^{-1}\left(u h_{1}-w h_{3}\right)+2 v f_{1} h_{3} g_{2 x}+v_{x} f_{1} g_{2} h_{3}> \\
& +<g_{0}, f_{1}\left(h_{3 x x x}-h_{3 x}\right)+f_{3}\left(h_{1 x}-h_{1 x x x}\right)+3 f_{1 x}\left(h_{3 x x}-h_{3}\right)+3 f_{3 x}\left(h_{1}-h_{1 x x}\right)> \\
& -(f \leftrightarrow h)+\mathrm{c} \cdot \mathrm{p} . \\
= & <2 v g_{2 x} f_{1} h_{3}+f_{1} v_{x} g_{2} h_{3}-2 f_{3} g_{2 x} h_{1} v-f_{3} v_{x} g_{2} h_{1}>+<g_{0},\left(\partial^{3}-4 \partial\right)\left(f_{1} h_{3}-f_{3} h_{1}\right)> \\
& +\frac{3}{2}<u h_{1}-w h_{3}, f_{3 x} g_{1}-f_{1} g_{3 x}+f_{1 x} g_{3}-f_{3} g_{1 x}>+\mathrm{c} \cdot \mathrm{p} . \\
= & <2 v g_{2 x} f_{1} h_{3}+f_{1} v_{x} g_{2} h_{3}-2 v g_{2 x} h_{1} f_{3}-f_{3} v_{x} g_{2} h_{1}> \\
& +\frac{3}{2}<u h_{1}-w h_{3}, f_{3 x} g_{1}-f_{1} g_{3 x}+f_{1 x} g_{3}-f_{3} g_{1 x}> \\
& +\frac{1}{2}<f_{3} h_{1}-f_{1} h_{3}, 3 u g_{1 x}+u_{x} g_{1}+2 v_{x} g_{2}+4 v g_{2 x}+3 w g_{3 x}+g_{3} w_{x}> \\
= & \frac{3}{2}<u h_{1}-w h_{3}, f_{3 x} g_{1}-f_{1} g_{3 x}+f_{1 x} g_{3}-f_{3} g_{1 x}> \\
& +\frac{1}{2}<f_{3} h_{1}-f_{1} h_{3}, 3 u g_{1 x}+u_{x} g_{1}+3 w g_{3 x}+g_{3} w_{x}>+\mathrm{c} \cdot \mathrm{p} . \\
= & \frac{1}{2}<u, 3 h_{1}\left(f_{3 x} g_{1}-f_{1} g_{3 x}+f_{1 x} g_{3}-f_{3} g_{1 x}\right)+3\left(f_{3} h_{1}-f_{1} h_{3}\right) g_{1 x}-\left(\left(f_{3} h_{1}-f_{1} h_{3}\right) g_{1}\right)_{x}> \\
& +\frac{1}{2}<w, 3 h_{3}\left(f_{1} g_{3 x}+f_{3} g_{1 x}-f_{3 x} g_{1}-f_{1 x} g_{3}\right)+3 g_{3 x}\left(f_{3} h_{1}-f_{1} h_{3}\right) \\
& -\frac{1}{2}<w,\left(\left(f_{3} h_{1}-f_{1} h_{3}\right) g_{3}\right)_{x}>+\mathrm{c} . \mathrm{p} . \\
= & 0 .
\end{aligned}
$$

Therefore, the Hamiltonian operators $\mathscr{J}$ and $\mathscr{K}$ are compatible and the proof of the theorem is completed.

According to the bi-Hamiltonian theory, the operator $\mathscr{R}$ is a recursion operator with hereditary property, which may be used to generate a hierarchy of commuting flows. 
N.H. Li \& Q.P. Liu

\section{Acknowledgments}

This work is supported by the National Natural Science Foundation of China (grant numbers: 10971222 and 11271366) and the Fundamental Research Funds for Central Universities.

\section{References}

[1] R. Beals, D. Sattinger and J. Szmigielski, Acoustic scattering and the extended Korteweg-de Vries hierarchy, Adv. Math. 140 (1998) 190-206.

[2] A. Boutet de Monvel, A. Kostenko, D. Shepelsky and G. Teschl, Long-time asymptotics for the Camassa-Holm equation, SIAM J, Math. Anal. 41 (2009) 1559-1588.

[3] R. Camassa and D. D. Holm, An integrable shallow water equation with peaked solitons, Phys. Rev. Lett. 71 (1993) 1661-1664.

[4] M. Chen, S. Q. Liu and Y. J. Zhang, A 2-component generalization of the Camassa-Holm equations and its solitons, Lett. Math. Phys. 75 (2006) 1-15.

[5] A. Constantin, On the inverse spectral problem for the Camassa-Holm equation, J. Funct. Anal. 155 (1998) 352-363.

[6] A. Constantin, On the scattering problem for the Camassa-Holm equation, Proc. R. Soc. London A 457 (2001) 953-970.

[7] A. Constantin, The trajectories of particles in Stokes waves, Invert. Math. 166 (2006) 523-535.

[8] A. Constantin and J. Escher, Wave breaking for nonlinear nonlocal shallow water equations, Acta Math. 181 (1998) 229-243.

[9] A. Constantin and J. Escher, Particle trajectories in solitary water waves, Bull. Amer. Math. Soc. 44 (2007) 423-431.

[10] A. Constantin and J. Escher, Analyticity of periodic traveling free surface water waves with vorticity, Ann. Math. 173 (2011) 559-568.

[11] A. Constantin and R. I. Ivanov, On an integrable two-component Camassa-Holm shallow water system, Phys. Lett. A 372 (2008) 7129-7132.

[12] A. Constantin, T. Kappeler, B. Kolev and P. Topalov, On geodesic exponential maps of the Virasoro group, Ann. Global Anal. Geom. 31 (2007) 155-180.

[13] A. Constantin and B. Kolev, Geodesic flow on the diffeomorphism group of the circle, Comment. Math. Helv. 78 (2003) 787-804.

[14] A. Constantin and D. Lannes, The hydrodynamical relevance of the Camassa-Holm and DegasperisProcesi equations, Arc. Rat. Mech. Anal. 192 (2009) 165-186.

[15] A. Constantin and H. P. McKean, A shallow water equation on the circle, Commun. Pure Appl. Math. 52 (1999) 949-982.

[16] A. Constantin and W. Strauss, Stability of peakons, Commun. Pure Appl. Math. 53 (2000) 603-610.

[17] A. Constantin, V. S. Gerdjikov and R. I. Ivanov, Inverse Scattering transformation for the CamassaHolm equation. Inverse Problems 22 (2006) 2197-2207.

[18] L. Fontanelli, P. Lorenzoni and M. Pedroni, A three-component extension of the Camassa-Holm hierarchy, Lett. Math. Phys. 78 (2006) 125-137.

[19] B. Fuchssteiner, Some tricks from the symmetry-toolbox for nonlinear equations: generalizations of the Camassa-Holm equation, Physica D 95 (1996) 229-243.

[20] B. Fuchessteiner and A. S. Fokas, Symplectic structures, their Bäcklund transformation and hereditary symmetries, Physica D 4 (1981) 47-66.

[21] X. G. Geng and B. Xue, A three-component generalization of Camassa-Holm equation with N-peakon solutions, Adv. Math. 226 (2011) 827-839.

[22] F. Gesztesy and H. Holden, Algebro-geometric solutions of the Camassa-Holm hierarchy, Rev. Mat. Iberoamericana 19 (2003) 73-142.

[23] A. N. W. Hone and J. P. Wang, Integrable peakon equations with cubic nonlinearity, J. Phys. A: Math. Theor. 41 (2008) 372002.

[24] D. Ionescu-Kruse, Variational derivation of the Camassa-Holm shallow water wave equation, J. Nonlinear Math. Phys. 14 (2007) 311-320. 
[25] R. I. Ivanov, Water waves and integrability, Philos. Trans. R. Soc. London A 365 (2007) 2267-2280.

[26] R. S. Johnson, Camassa-Holm, Korteweg-de Vries and related models for water waves, J. Fluid Mech. 455 (2002) 63-82.

[27] R. S. Johnson, On solutions of the Camassa-Holm equation, Proc. R. Soc. Lond. A 459 (2003) 16871708.

[28] B. Kolev, Bi-Hamiltonian systems on the dual of the Lie algebra of vector fields of the circle and periodic shallow water equations, Philos. Trans. R. Soc. London A 365 (2007) 2333-2357.

[29] S. Kouranbaeva, The Camassa-Holm equation as a geodesic flow on the diffeomorphism group, J. Math. Phys. 40 (1999) 857-868.

[30] J. Lenells, The scattering approach for the Camassa-Holm equation, J. Nonlinear Math. Phys. 9 (2002) 389-393.

[31] J. Lenells, A variational approach to the stability of periodic peakons, J. Nonlinear Math. Phys. 11 (2004) 151-163.

[32] J. Lenells, The correspondence between KdV and Camassa-Holm, IMRN (International Mathematical Research Notices) 71 (2004) 3797-3811.

[33] Y. S. Li and J. E. Zhang, The multi-soliton solution of the Camassa-Holm equation, Proc. R. Soc. Lond. A 460 (2004) 2617-2627.

[34] Y. Matsuno, Parametric representation for the multisoliton solution of the Camassa-Holm equation, $J$. Phys. Soc. Japan. 74 (2005) 1983-1987.

[35] G. Misiolek, A shallow water equation as a geodesic flow on the Bott-Virasoro group, J. Geom. Phys. 24 (1998) 203-208.

[36] P. J. Olver and P. Rosenau, Tri-Hamiltonian duality between solitons and compactons, Phys. Rev. E 53 (1996) 1900-1906.

[37] Z. J. Qiao, The Camassa-Holm hierarchy, $N$-dimensional integrable systems, and algebro-geometric solution on a symplectic manifold, Commun. Math. Phys. 239 (2003) 309-341.

[38] J. F. Toland, Stokes Waves, Topol. Methods Nonlinear Anal. 7 (1996) 1-48.

[39] G. B. Whitham, Linear and Nonlinear Waves (Wiley-Interscience, New York, 1974). 